\title{
One-Dimensional Mott Insulator Formed by Quantum Vortices in Josephson Junction Arrays
}

\author{
Alexander van Oudenaarden and J.E. Mooij \\ Department of Applied Physics and Delft Institute of Micro-electronics and Submicron-technology (DIMES), \\ Delft University of Technology, P.O. Box 5046, 2600 GA Delft, The Netherlands
}

(Received 26 February 1996)

\begin{abstract}
The quantum transport of vortices in very long and narrow arrays of small Josephson junctions has been studied experimentally. The chemical potential of the system can be changed by an external magnetic field. When the vortex density is commensurate with the one-dimensional junction lattice, the vortex mobility vanishes for a finite window of the magnetic field. The localization of the vortices is due to the forming of a one-dimensional Mott insulator. [S0031-9007(96)00521-2]
\end{abstract}

PACS numbers: 74.50.+r, 05.30.Jp

Low-dimensional systems of strongly interacting bosons reveal a broad spectrum of quantum phase transitions. For bosons with repulsive interactions in a periodic potential, a phase transition occurs from a superfluid to a Mott insulator when the boson density changes from incommensurate to commensurate with the periodic lattice [1]. This phase transition is driven by the competition between the hopping of bosons and the repulsive interactions between the bosons. In two dimensions, the transition has been studied in experimental systems such as ${ }^{4} \mathrm{He}$ on graphite [2] and flux lines in superconductors with artificial pinning centers [3]. The phase diagram of one-dimensional bosons on a lattice has been theoretically studied with analytical [4] and quantum Monte Carlo methods [5]. The theoretical studies focus mainly on interacting bosons with short-range interactions. Experimentally, Josephson junction arrays can be used to study interacting bosons in one dimension. We do not know of any other experimental one-dimensional boson system.

A voltage across a Josephson junction array is connected with the motion of vortices. Vortices in Josephson junction arrays behave as mass-carrying particles in a periodic potential landscape caused by the junction lattice. Traveling from cell to cell the vortex has to overcome an energy barrier, which is proportional to the Josephson coupling energy $E_{J}$. The vortex mass is inversely proportional to the charging energy $E_{C}$ [6]. In the limit of high barriers and large vortex mass $\left(E_{J} \gg E_{C}\right)$ the vortices behave as classical particles. For smaller mass and lower barrier height $\left(E_{J} \approx E_{C}\right)$ the vortices exhibit quantum mechanical properties [6,7]. These quantum vortices behave as bosons. They repel each other over a long range with a force proportional to $E_{J}$. Because of the quantum mechanical nature of the vortices and the periodic lattice potential, bands will form. The bandwidth is proportional to $E_{C}$. Josephson junction arrays have the advantage that $E_{J}$ and $E_{C}$ are experimentally well controllable and can be varied over a wide range.

Is this Letter, we report the observation of a onedimensional Mott insulator formed by quantum vortices in a very long and narrow array of Josephson junctions.
Around one-dimensional commensurate densities the mobility of the vortices vanishes for a finite window of the magnetic field. By varying $E_{J} / E_{C}$ the stability of the Mott insulator can be changed. In this way the lobelike shape of the Mott insulating phase of interacting bosons in a one-dimensional periodic potential has been determined experimentally for the first time.

The samples consist of an array of Josephson junctions. Each island is coupled to four neighboring islands (square cell) with small Josephson junctions. The length $L$ of the array ( $L=1000$ cells) is much larger than the width $W$ of the array ( $W=7$ cells). Along the length of the array the islands at both edges are connected by a narrow superconducting strip (busbar). These busbars repel the vortices and confine the vortex motion to one dimension. The current is injected in the middle and the voltage probes are situated at one end of the busbar. The sample layout is schematically sketched in Fig. 1. The area of a cell $S$ is $2 \mu \mathrm{m}^{2}$.

Samples were fabricated using electron beam lithography and a shadow evaporation technique. They consist of high quality $\mathrm{Al}-\mathrm{Al}_{2} \mathrm{O}_{3}-\mathrm{Al}$ tunnel junctions. We present the results of three different samples, which are indicated by $\mathrm{A}, \mathrm{B}$, and $\mathrm{C}$. The capacitances $C$, estimated from the

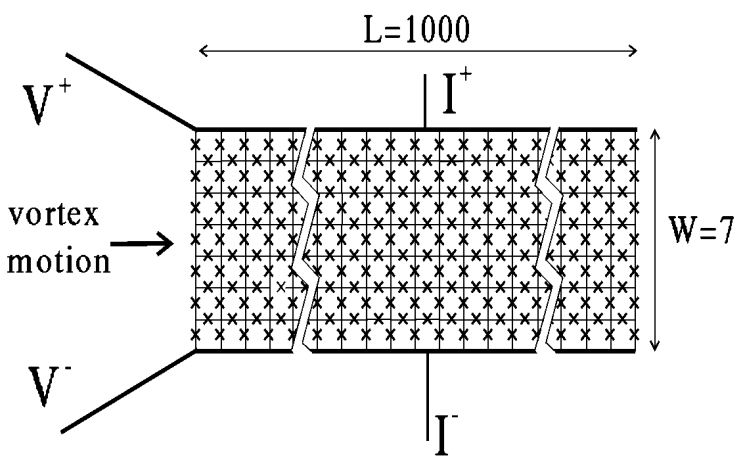

FIG. 1. Layout of the sample. The transport properties are determined by a four-terminal measurement. The current is injected in the middle of the sample and the resulting voltage is measured at one end of the sample. The Josephson junctions are denoted by crosses. 
overlap area of the junctions, are 2.1 (A), 1.5 (B), and 1.0 $\mathrm{fF}(\mathrm{C})$, which correspond to values of the charging energy $E_{C}=e^{2} / 2 C$ of $0.44(\mathrm{~A}), 0.70(\mathrm{~B})$, and $0.93 \mathrm{~K}(\mathrm{C})$. The normal state junction resistances $r_{n}$ are $5.6(\mathrm{~A}), 7.6(\mathrm{~B})$, and $11.1 \mathrm{k} \Omega(\mathrm{C})$. The variations in $C$ and $r_{n}$ over one array are approximately $5 \%$. The variations are estimated by resistance measurements on separate test circuits next to the arrays and by scanning electron microscopy photographs. The Josephson coupling energy $E_{J}$ is given by $i_{c 0} \Phi_{0} / 2 \pi$, where $i_{c 0}$ is the intrinsic junction critical current and $\Phi_{0}$ is the flux quantum. The Ambegaokar-Baratoff result for aluminum $\left(i_{c 0} r_{n}=300 \mu \mathrm{V}\right)$ leads to $E_{J} / E_{C}$ ratios of 2.8 (A), 1.3 (B), and 0.7 (C). In the absence of geometrical inductance effects the interaction range of the vortex is not screened and the interaction range is cut off by the sample size. In the presence of induced magnetic fields the interaction range is screened. For a two-dimensional array [8] the interaction range is $\lambda_{2 \mathrm{D}}=\Phi_{0} / 2 \pi \mu_{0} i_{c 0} a(\gg L)$, where $a$ is the lattice spacing. The interaction range for a one-dimensional parallel Josephson junction array [9] is $\lambda_{1 \mathrm{D}}=\sqrt{\lambda_{2 \mathrm{D}}}(60-80$ cells $)$. The interaction range in a very long and narrow array is bounded by these two limits. Therefore the long-range interaction between the vortices plays an important role.

The experiments were performed in a dilution refrigerator. Electrical signals were filtered by $R C$ and microwave filters at the temperature of the mixing chamber. The leads were additionally filtered by feedthrough rfi filters at room temperature. Magnetic interference was screened by a superconducting shield inside a Mumetal box. A magnetic field was applied by two small superconducting coils in the Helmholtz configuration. The two-dimensional frustration $f$ is defined as the applied magnetic flux $B S$ per cell area divided by $\Phi_{0}$. The one-dimensional frustration $n$, relevant for the physics of the one-dimensional system, is equal to $W B S / \Phi_{0}=W f$. For an infinite system $n$ equals the one-dimensional vortex density.

The zero-bias resistance $R_{0}$ of the arrays was measured by a lock-in technique as a function of the applied magnetic field. The maximum bias current was $10 \mathrm{pA}$ per junction, which was small enough to determine the resistance in the linear response regime. In Fig. 2 $R_{0}$ normalized to the normal state array resistance $R_{n}$ $\left(=W r_{n} / L\right)$ is shown as a function of the one-dimensional frustration $n$ for sample C. The zero-bias resistance increases with increasing $n$. At $n=1 / 3$ the resistance drops sharply to zero. We observed the same dip at $n=2 / 3$. We could not observe a decrease of $R_{0}$ at $n=1 / 2$, which is remarkable. In other samples with $E_{J} / E_{C} \approx 5$, we have observed sharp maxima in the depinning current at $n=2 / 3,1,3 / 2,2$, and 3 . We will not discuss those samples here. In the inset of Fig. 2 the $I-V$ characteristics for a commensurate $(n=$ $1 / 3)$ and an incommensurate $(n=0.340)$ vortex density are shown. At the commensurate density, the zerobias resistance is zero (the vortices are localized) and

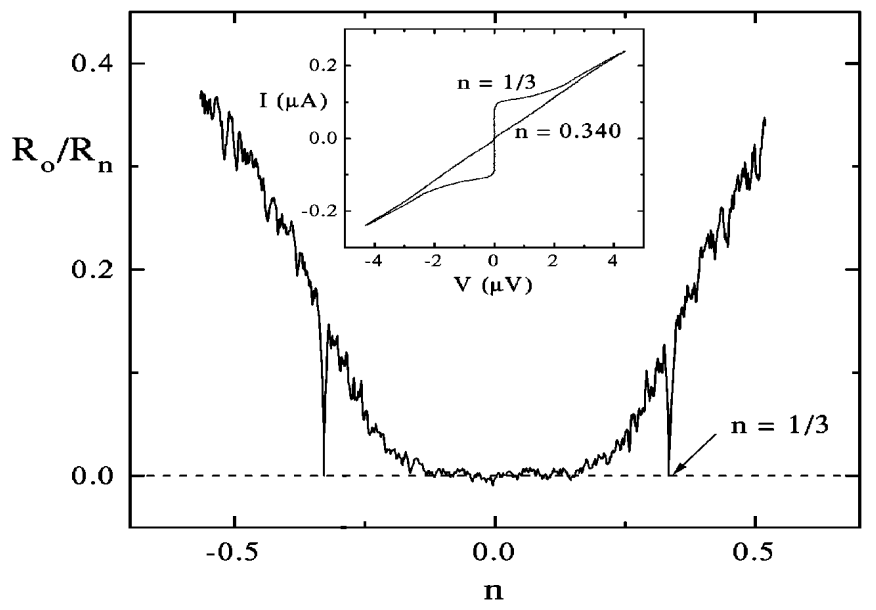

FIG. 2. The normalized resistance $R_{0} / R_{n}$ as a function of the one-dimensional frustration $n$ for sample $\mathrm{C}(T=30 \mathrm{mK})$. The resistance drops sharply to zero at the one-dimensional commensurate state $n=1 / 3$. In the inset the $I-V$ characteristics are shown for a commensurate filling $n=1 / 3$ and an incommensurate filling $n=0.340$ for sample C.

the depinning current is $0.1 \mu \mathrm{A}\left(0.04 i_{c 0}\right)$, whereas for incommensurate filling the zero-bias resistance has a finite value (vortices are delocalized) and the depinning current is 2 orders of magnitude smaller than for the commensurate density. In the case of localized vortices $\left(R_{0}=0\right)$ the $I-V$ characteristic exhibits superconducting properties.

In Fig. 3, a blowup of the $n=1 / 3$ dip is shown for three different samples. The figure shows that the window where $R_{0}=0$ decreases as $E_{J} / E_{C}$ decreases. Adjacent to the dip the resistance oscillates as a function of the magnetic field with a period $\delta n \approx 1 / 1000=1 / L$. This oscillating behavior is a finite size effect. Each period corresponds to adding an extra vortex to the array [10]. Both the oscillating resistance and the sharpness of the dips at the one-dimensional commensurate values are indicative for the high quality of the samples.

The experimental findings can be interpreted using a model of interacting bosons on a periodic lattice [1]. The competition between the repulsive interaction strength, proportional to $E_{J}$, and the bandwidth, proportional to $E_{C}$, determines the mobility of the vortices. The onedimensional frustration $n$ can be associated with the chemical potential. Around the one-dimensional commensurate filling the vortices are localized $\left(R_{0}=0\right)$ : The vortices form a Mott insulator. In this phase the potential energy required to add or remove a vortex is larger than the energy the system gains when it allows a vortex to hop through the one-dimensional lattice. In the Mott insulating phase, the number of vortices is locked to the commensurate value. An energy gap $E_{g}$ for the creation of a particle or hole excitation exists in the Mott insulating phase. Precisely at the commensurate density, 


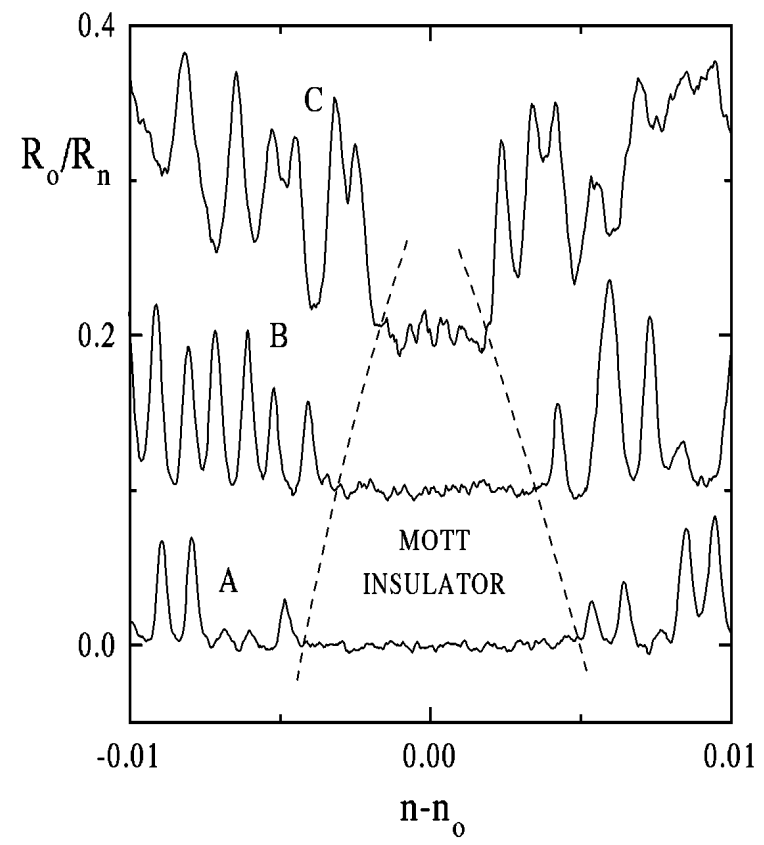

FIG. 3. The normalized resistance $R_{0} / R_{n}$ as a function of the one-dimensional frustration $n$ for samples $\mathrm{A}, \mathrm{B}$, and $\mathrm{C}$ $(T=30 \mathrm{mK})$ around the one-dimensional commensurate state $n_{0}=1 / 3$. The curves for samples $\mathrm{B}$ and $\mathrm{C}$ have an offset in $R_{0} / R_{n}$ of 0.1 and 0.2 , respectively, which is proportional to the relative increase of $E_{C} / E_{J}$ for samples B and C. In this way the phase diagram can be constructed. The dashed lines indicate the phase boundary between the conducting phase and the Mott insulating phase.

$E_{g}$ has its maximum value. The energy gap decreases away from the commensurate filling and equals zero at the phase boundary. At incommensurate filling, the kinetic energy gain is larger than the potential energy cost to add or remove a vortex, which results in delocalized vortices $\left(R_{0} \neq 0\right)$. Decreasing $E_{J} / E_{C}$ results in a decrease of the interaction strength and an increase of the bandwidth. Therefore, for samples with relatively large $E_{J} / E_{C}$ the vortices are localized for a larger range of the magnetic field than samples with a relatively small $E_{J} / E_{C}$. In this way the lobelike shape of the phase diagram in Fig. 3 can be understood.

In Fig. 4 the range $\Delta n$ where the Mott insulator is stable $\left(R_{0}=0\right)$ is plotted as a function of temperature. The shape of the lobe near the phase boundary can be deduced from the temperature dependence. At finite temperature the thermal activation energy can overcome $E_{g}$. When the temperature is increased, the first instability will occur near the phase boundary, because here $E_{g}$ is smallest. The phase boundaries move towards $n_{0}$ when the temperature is increased. Experimentally we have observed a linear decrease of $\Delta n$ as a function of temperature for $T<500 \mathrm{mK}$. This linear dependence indicates a linear relation between $E_{g}$ and $n-n_{0}$ near the phase boundaries. The slope of the $\Delta n$ versus $T$

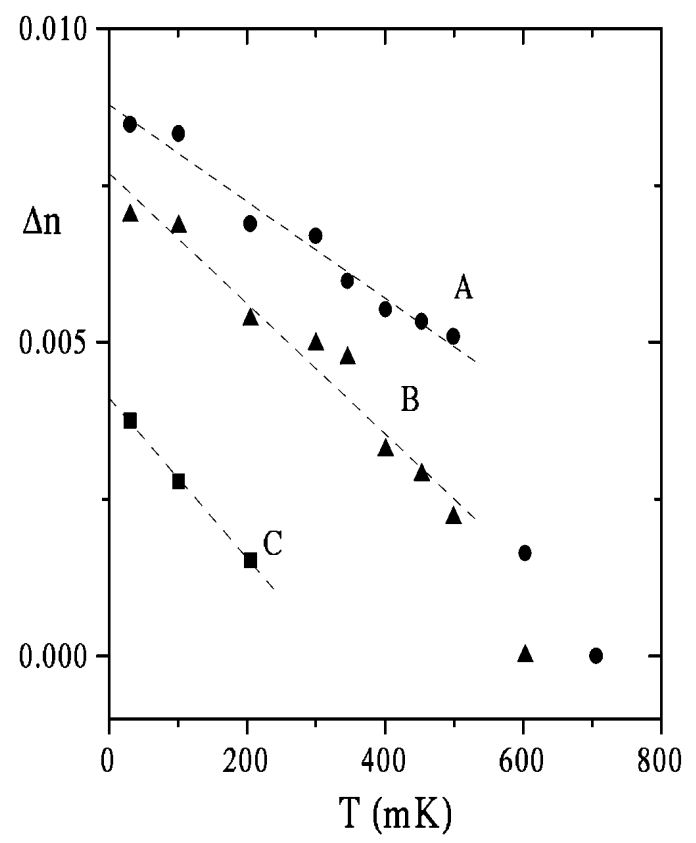

FIG. 4. The range $\Delta n$ for which the vortices form a Mott insulator as a function of the temperature. Note that $\Delta n$ is of the order of $10^{-2}$, which corresponds to a magnetic induction $B$ of only $10^{-2} \mathrm{G}$.

curve is approximately the same for the three samples. For $T>500 \mathrm{mK}, \Delta n$ drops sharply to zero. For these high temperatures the influence of quasiparticles becomes important, which suppresses quantum effects and provides a dissipation mechanism for the vortices. At higher temperatures the superconducting gap of aluminum also starts to decrease, which results in a decrease of $E_{J}$.

In conclusion, we have experimentally observed a onedimensional Mott insulator formed by quantum vortices in very long and narrow arrays of small Josephson junctions. We have investigated the stability of the Mott insulating phase as a function of the repulsion between the vortices $\left(\propto E_{J}\right)$, the bandwidth $\left(\propto E_{C}\right)$, and the temperature. The experiments indicate that long and narrow arrays are promising experimental systems to study interacting bosons in one dimension.

We want to thank A. A. Odintsov, A. van Otterlo, K.-H. Wagenblast, R. Fazio, H. S. J. van der Zant, P. Hadley, and S.J.K. Várdy for useful discussions. The Delft Institute of Micro-electronics and Submicrontechnology (DIMES) is acknowledged for the support in fabrication. The work was supported by the Dutch Foundation for Fundamental Research on Matter (FOM).

[1] M.P. A. Fisher, P. B. Weichman, G. Grinstein, and D. S. Fisher, Phys. Rev. B 40, 546 (1989).

[2] G. T. Zimanyi, P. A. Crowell, R. T. Scalettar, and G. G. Batrouni, Phys. Rev. B 50, 6515 (1994), and references therein. 
[3] M. Baert, V.V. Metlusho, R. Jonckheere, V. V. Moshchalkov, and Y. Bruynseraede, Phys. Rev. Lett. 74, 3269 (1995); K. M. Beauchamp, T.F. Rosenbaum, U. Welp, G. W. Crabtree, and V.M. Vinokur, Phys. Rev. Lett. 75, 3942 (1995).

[4] J. K. Freericks and H. Monien, Europhys. Lett. 26, 545 (1994); Phys. Rev. B 53, 2691 (1996).

[5] G. G. Batrouni, R. T. Scalettar, and G. T. Zimanyi, Phys. Rev. Lett. 65, 1765 (1990); P. Niyaz, R. T. Scalettar, C. Y. Fong, and G. G. Batrouni, Phys. Rev. B 50, 362 (1994), and references therein.

[6] E. Simanek, Solid State Commun. 48, 1023 (1983); S. E. Korshunov, Physica (Amsterdam) 152B, 261 (1988); A. I. Larkin, Yu. N. Ovchinikov, and A. Schmid, Phys- ica (Amsterdam) 152B, 266 (1988); U. Eckern and A. Schmid, Phys. Rev. B 39, 6441 (1989).

[7] H. S. J. van der Zant, F. C. Fritschy, W. J. Elion, and J. E. Mooij, Phys. Rev. Lett. 69, 2971 (1992); W. J. Elion, J. J. Wachters, L. L. Sohn, and J. E. Mooij, Phys. Rev. Lett. 71, 2311 (1993).

[8] J. R. Phillips, H.S. J. van der Zant, J. White, and T.P. Orlando, Phys. Rev. B. 47, 5219 (1993).

[9] R. D. Bock, J. R. Phillips, H. S. J. van der Zant, and T. P. Orlando, Phys. Rev. B. 49, 10009 (1994).

[10] A. van Oudenaarden, S.L. Yeung, and J.E. Mooij, in Macroscopic Quantum Phenomena and Coherence in Superconducting Networks, edited by C. Giovanella and M. Tinkham (World Scientific, Singapore, 1995). 